\title{
On the formation of macrovoids in PMMA membranes
}

\author{
Juin-Yih Lai ${ }^{\mathrm{a}}$, Fung-Ching Lin ${ }^{\mathrm{a}}$, Tian-Tsair Wu ${ }^{\mathrm{a}}$, Da-Ming Wang ${ }^{\mathrm{b}, *}$ \\ a Membrane Research Laboratory, Department of Chemical Engineering, Chung Yuan University, Chung Li 32023, Taiwan \\ ${ }^{\mathrm{b}}$ Department of Chemical Engineering, National Taiwan University, Tapei 10617, Taiwan
}

Received 18 August 1997; received in revised form 27 July 1998; accepted 11 September 1998

\begin{abstract}
In this work, the formation of macrovoids in two membrane formation systems was investigated. The polymer and the coagulant used in these two systems were the same: PMMA and water, but the solvents used to dissolve PMMA were different - NMP for one system (system A) and acetone/Tween 80 solution for the other (system B). According to SEM and AFM analyses, it was found that, although the macrostructures of the membranes fabricated by systems A and B are similar, the microstructures are different. On the basis of optical observation of the growth of macrovoids, it is proposed that the difference in the microstructure can be explained by the difference in the growth mechanism of macrovoids: the growth of macrovoids is by convection for system A and by diffusion for system B. The difference in the growth mechanism of macrovoids can also account for some other differences between systems A and B: system B is more sensitive to the casting thickness than system $\mathrm{A}$, and the addition of salt in the coagulation bath can effectively inhibit the growth of macrovoids in system B but not in system A. (C) 1999 Elsevier Science B.V. All rights reserved.
\end{abstract}

Keywords: Membrane preparation; Membrane structure; Macrovoid; Formation mechanism

\section{Introduction}

Macrovoids, large elongated pores, can often be observed in the asymmetric polymeric membranes prepared by the phase inversion method [1]. The existence of macrovoids could jeopardize the mechanical integrity of membranes and result in compaction or collapse of membranes when being applied to high pressure processes [2]. On the other hand, the macrovoid structure is suitable for ultrafiltration process and can be employed as support layers for composite membranes [1]. Recent researches indicate that mem-

\footnotetext{
*Corresponding author. Tel.: +8862-23660433; fax: +886223623040; e-mail: daming@ccms.ntu.edu.tw
}

branes with macrovoid structure are useful in osmotic drug delivery and transdermal delivery systems [3,4]. Therefore, the formation mechanism of macrovoids has drawn a lot of research attentions [1,2,5-13]. For more detailed review of the development in this field, one can refer to the works of Smolders et al. [1] and Paulsen et al. [13].

Some researchers proposed that the growth of macrovoids during membrane formation is due to the convective flow induced by surface tension gradient [6] or by shrinkage of polymer matrix [2,5]. Others believed that the diffusional flow of solvent from the polymer solution surrounding the initiated macrovoids is responsible for the growth of macrovoids [1,9-12]. The main purpose of this work is to 
add new information in this respect. The data which will be shown below indicate that both convective and diffusional flows could result in the growth of macrovoids.

Poly(methyl methacrylate) (PMMA) was chosen as the model polymer because it is amorphous, which can prevent the membrane formation being complicated by crystallization. It was reported [14] that the membrane fabricated by immersing PMMA/NMP solution in water bath possessed macrovoid structure. Besides, it was shown that membranes with macrovoids can be obtained by adding Tween 80 (a hydrophilic surfactant) into the PMMA/acetone solution and immersing the resulted solution in a water bath [15]. In this work, the formation of macrovoids in these two membrane formation systems is studied. It will be shown that, although the macrostructures of the membranes fabricated by these two systems are similar, the microstructures, including the membrane surface structure and the structure of the wall of macrovoids, are different. A possible explanation for this observation will also be discussed: the difference in microstructure could be accounted for by the difference in the growth mechanism of macrovoids: one system is due to diffusion and the other is convection.

\section{Experimental}

\subsection{Materials}

Poly(methyl methacrylate) (PMMA) used in this study was supplied by Aldrich. The number averaged molecular weight is $140000 \mathrm{~g} / \mathrm{mol}$ (measured by GPC) and the DSC test was performed to confirm that the PMMA used is amorphous. The nonsolvents and solvents were of reagent grade and used without further purification. Distilled water was used throughout this study.

\subsection{Membrane preparation}

Two membrane formation systems were used to fabricated asymmetric PMMA membranes. In one system, PMMA was dissolved in NMP to form 12.5 vol\% casting solutions at room temperature. In the other system, the polymer solvent used was a solution of Tween 80 (18 vol\%) in acetone instead
Table 1

Comparison between systems A and B

\begin{tabular}{llll}
\hline & Polymer & Solvent & Coagulant \\
\hline System A & PMMA $^{\text {a }}$ & NMP & Water \\
System B & PMMA $^{\text {a }}$ & Acetone+Tween $80^{\text {b }}$ & Water \\
\hline
\end{tabular}

${ }^{a}$ Polymer composition in the casting solution is $12.5 \mathrm{vol} \%$.

b 18 vol\% of Tween 80 .

of NMP, and the PMMA concentration in the casting solution was also maintained at $12.5 \mathrm{vol} \%$. The polymer solutions were cast on a glass plate to a predetermined thickness of $300 \mu \mathrm{m}$ with a Gardner knife, and then immersed into a water bath for $10 \mathrm{~min}$. After precipitation, the membranes were peeled off and dried in air. For simplicity, from now on, the membrane formation system PMMA/NMP/water is denoted by system A and the other system, PMMA/ acetone + Tween $80 /$ water, by system $\mathrm{B}$. The difference between these two systems is summarized in Table 1.

\subsection{Determination of cloud-point curves}

Cloud-point curves were obtained by a simple titration method. Homogeneous PMMA casting solutions for systems A and B were prepared and water was then added slowly till the onset of turbidity. During titration, the solution was well stirred. The composition at the onset of turbidity, the so-called cloud point, represents the transition concentration between the one-phase and two-phase regions. The cloud-point curve can be interpreted as the envelope of the demixing region.

\subsection{SEM analysis}

The membrane structures were examined by a Hitachi (Model S570) scanning electron microscope (SEM). In SEM studies, membrane samples were fractured in liquid nitrogen and coated with gold to $\sim 150 \AA$.

\subsection{AFM analysis}

The microstructure of membrane surface was examined using a Digital Instrument (Model DI 5000) atomic force microscope (AFM). 


\subsection{Optical microscope studies}

The method used for the observation of macrovoid formation was similar to that developed by Strathmann et al. [5]. A drop of casting solution was placed between two microscope slides and a drop of coagulant (water), dyed with rhodamine B, was then introduced by a syringe. It should be noted that the dye, rhodamine B, was soluble in the solvents used in this study (NMP and acetone). The penetration fronts of water and rhodamine B into the casting solution were observed and videotaped under an Olympus BHT-M$113 \mathrm{D}$ optical microscope. The videotape was analyzed by a image processing software (Optimas 5.1, Bioscan) to determine the time dependence of the penetration distance of water.

\section{Results and discussion}

\subsection{Structures of the membranes prepared by systems $A$ and $B$}

The scanning electron micrographs of the membranes prepared by systems A and B are presented in Fig. 1(A) and (B). It can be seen that the membranes fabricated by these two systems possess similar macrostructures. Both membranes contain macro- voids extending over the whole cross-section. It was proposed that the formation of macrovoids is strongly related to the miscibility between the polymer solvent and the coagulant [10]. Our experimental results confirm this proposal: when $n$-hexane, less miscible with NMP and Tween 80, was used as the coagulant instead of water, macrovoids were obviously suppressed as shown in Fig. 2(A) and (B).

Although the membrane macrostructures shown in Fig. 1(A) and (B) are alike, the microstructures for systems $\mathrm{A}$ and $\mathrm{B}$, which will be presented in the following are in fact different. The microstructure of the membrane surface was examined using AFM and the results are shown in Fig. 3(A) and (B). It can be seen that, for both systems, the membrane surface is not smooth but consists of a lot of small bumps, which is believed to be polymer aggregates. Obviously, the number of bumps for system $B$ is much less than that for system A but the size of bumps is much larger, indicating that the small bumps (polymer aggregates) on the membrane surface for system B can coalesce more tightly than that for system A. In addition, the microstructure of the wall of macrovoids was examined by SEM. The scanning electron micrographs of the wall of macrovoids in the middle of membranes for systems A and B are presented in Fig. 4(A) and (C), and the microstructures of the wall of macrovoids near the bottom of membranes are illustrated in Fig. 4(B)

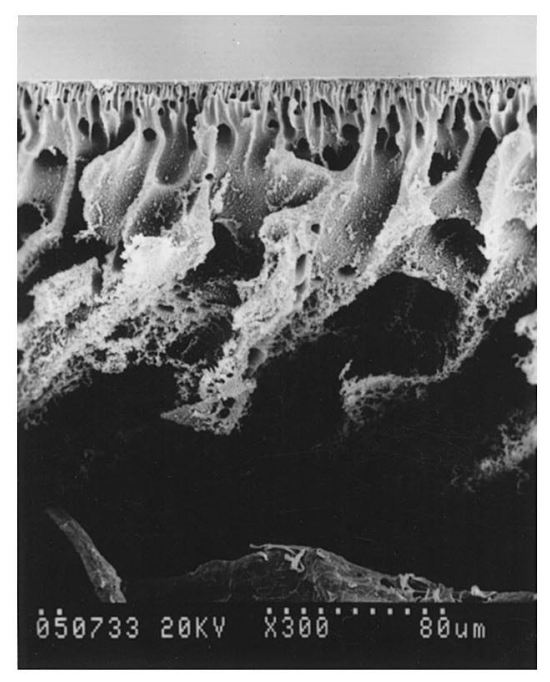

(a)

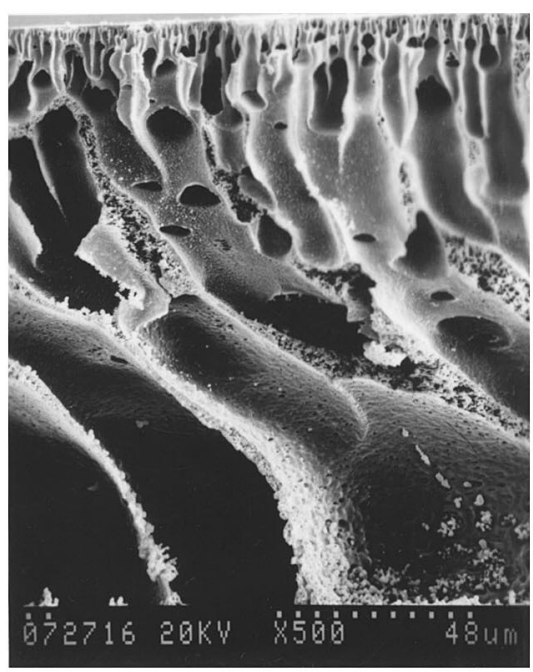

(b)

Fig. 1. SEM's of the membranes fabricated by systems A and B: (A) system A; (B) system B. 


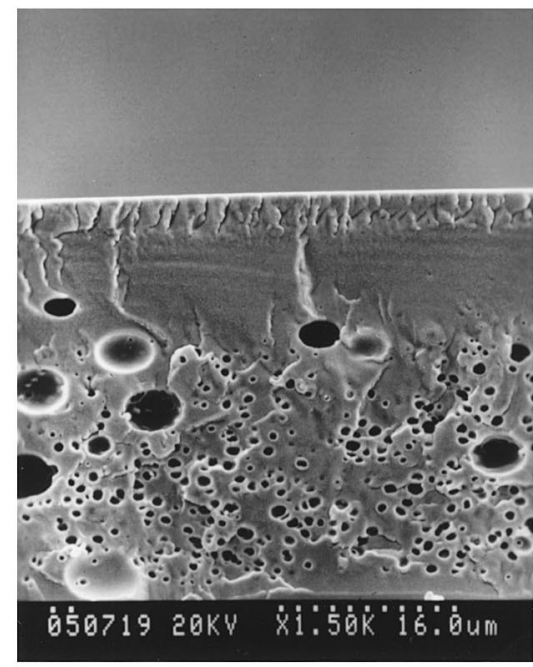

(a)

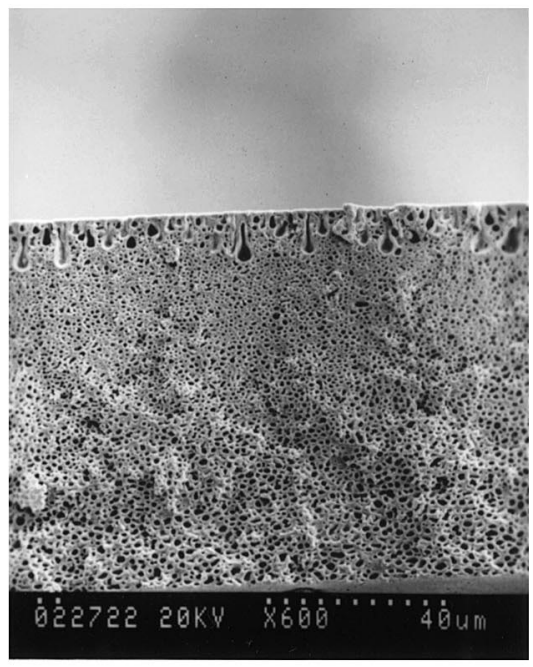

(b)

Fig. 2. SEM's of the membranes fabricated by systems A and B, but the coagulant water is replaced by $n$-hexane: (A) system A; (B) system B.

and (D). For system A, the wall of the macrovoids is composed of nodules (Fig. 4(A) and (B)). For system $\mathrm{B}$, the wall of macrovoids is smoother as shown in Fig. 4(C) and (D). An interesting point which should be noted is that open pores can be observed on the bottom wall of macrovoids for system B (Fig. 4(D)) but not for system A (Fig. 4(B)).

On basis of the above discussion, it is known that, although macrovoids can be observed for both systems $\mathrm{A}$ and $\mathrm{B}$ and the macrostructures are similar, the microstructures surrounding the macrovoids are in fact quite different. In the following sections, investigations will be presented which were carried out to account for the difference in microstructure between systems A and B.

\subsection{Thermodynamic and kinetic studies of the two membrane formation systems}

To understand the difference in microstructure between systems $\mathrm{A}$ and $\mathrm{B}$, the difference in the formation of macrovoids between these two systems was investigated. It is known that the formation of macrovoids is strongly related to the thermodynamic and kinetic behaviors of the membrane formation system [1]; therefore, thermodynamic and kinetic studies of these two systems were performed.
It was reported [1] that the behavior of liquid-liquid demixing plays an important role in membrane formation. The position of the binodal curve in a ternary phase diagram can be used to describe the liquidliquid demixing region. Larger demixing region indicates that the polymer solution is easier to phase separate [17]. In the present work, cloud-point curves were determined to represent the binodal curves. The measured cloud-point curves for systems A and B are depicted in Fig. 5. It can be seen that the two curves almost overlap, indicating that the two systems have similar demixing regions. Therefore, it seems that the difference in microstructures between the two systems cannot be explained by the difference in the demixing region.

It should be noted that, for polydisperse polymer systems, the cloud-point curve might not coincide with the binodal curve $[18,19]$. The PMMA used in this work is polydisperse polymer. Therefore, the cloud-point curves presented in Fig. 5 might differ from the binodal curves. However, the error caused by the negligence of the effect of polydispersity is roughly the same for systems $\mathrm{A}$ and $\mathrm{B}$, because, the polymer used is the same. Therefore, although the binodal curves would shift from the lines presented in Fig. 5 after considering the effect of polydispersity, both lines would shift similarly and still overlap. In other words, after the effect of polydispersity is taken 


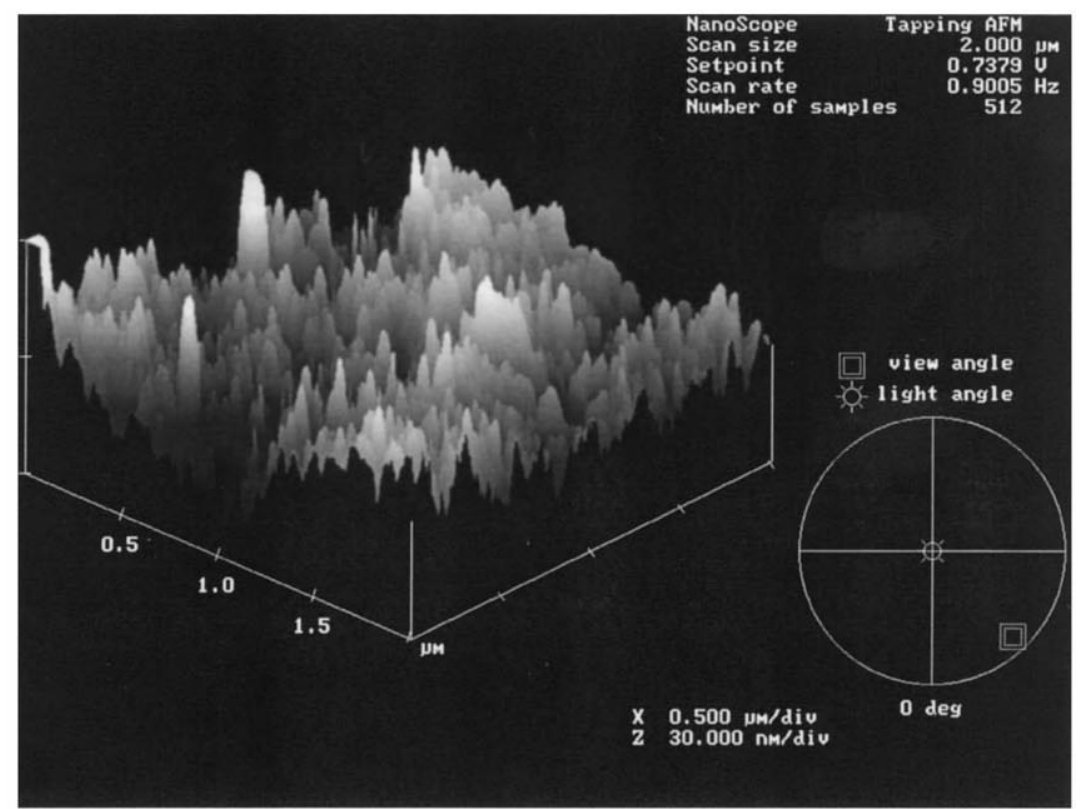

(a)

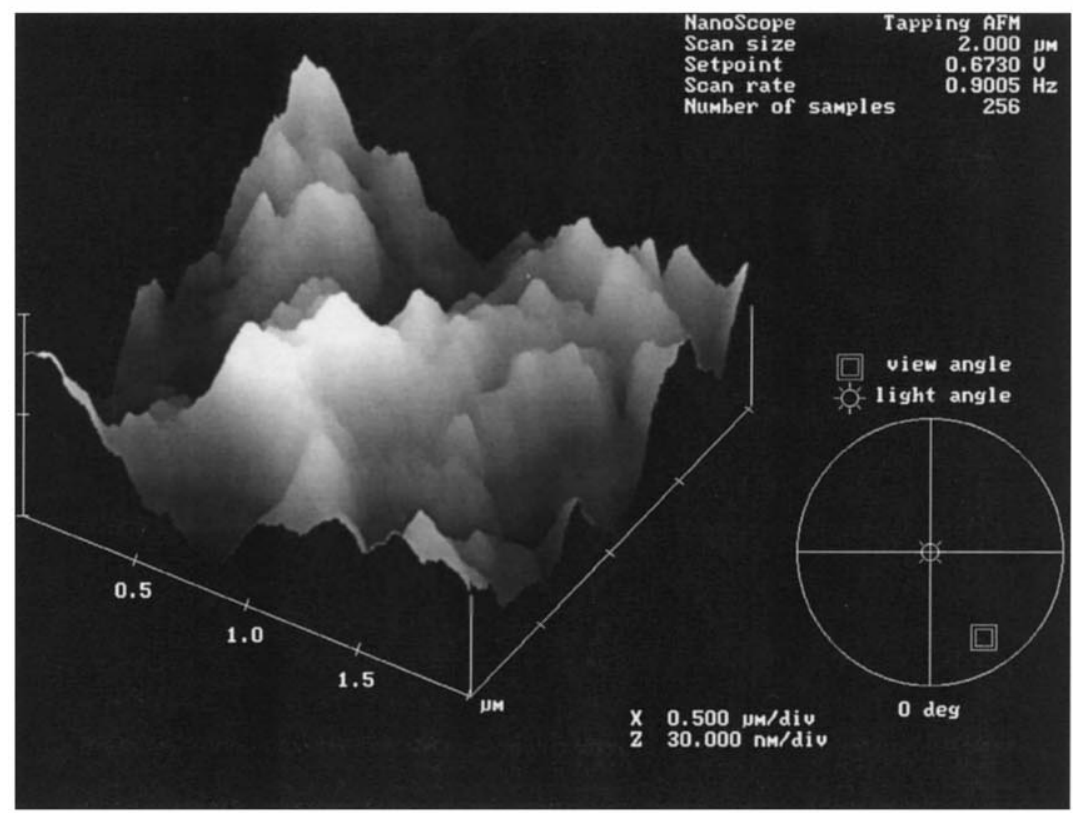

(b)

Fig. 3. AFM analysis on the membranes surface structure: (A) system A; (B) system B.

into account, the same conclusion that systems A and $\mathrm{B}$ have similar demixing regions would still be obtained.
To understand the kinetics of the formation of macrovoids, experiments of optical observation were performed. The formation of macrovoids was 


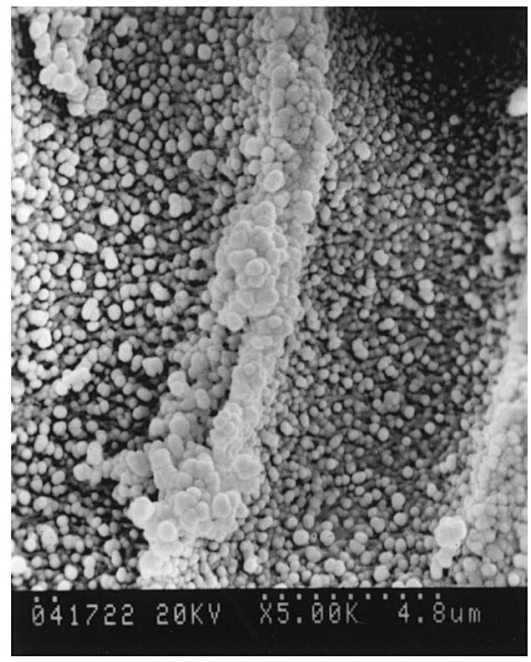

(a)

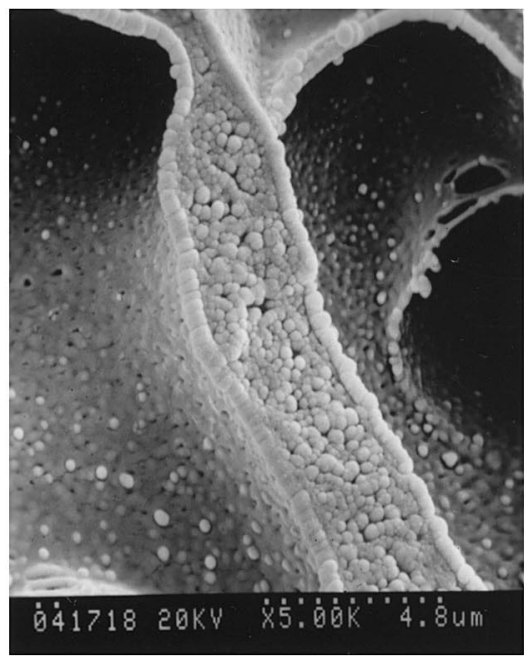

(c)

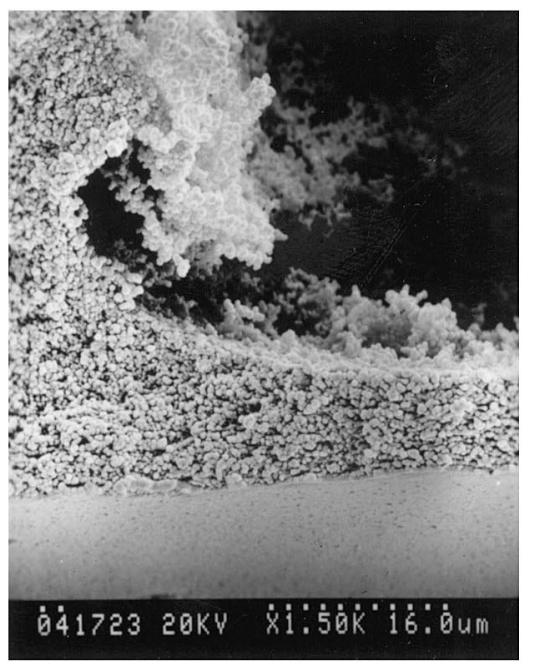

(b)

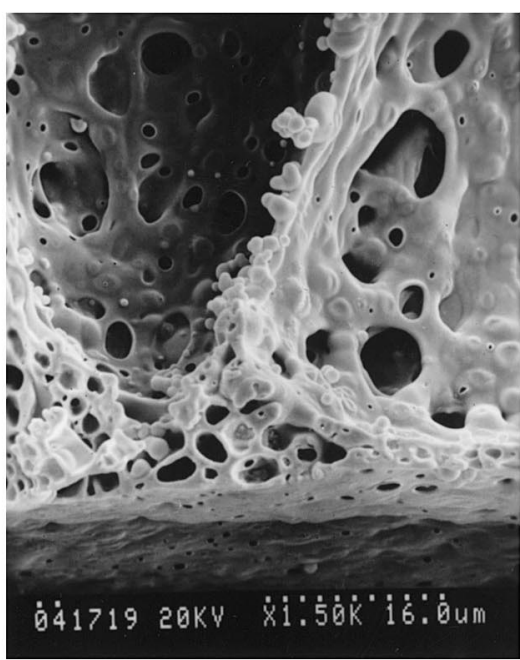

(d)

Fig. 4. Microstructures of the wall of macrovoids in systems A and B: (A) wall of macrovoids in the middle of membranes (system A); (B) wall of macrovoids near the bottom of membranes (system A); (C) wall of macrovoids in the middle of membranes (system B); (D) wall of macrovoids near the bottom of membranes (system B).

observed under a microscope following the procedures described in Section 2.6. Figs. 6 and 7 describe the penetration of water (coagulant) into the casting solution for systems A and B. The front of red color represents the penetration of rhodamine $\mathrm{B}$, a dye added in water, into the casting solution. And the other penetration front represents the growth of macrovoids resulted from the penetration of water.
For system A, immediately after the contact of water with the solution of PMMA/NMP, both water and rhodamine $\mathrm{B}$ can penetrate into the casting solution and the two fronts, front of water and front of dye, overlapped in the first few seconds as shown in Fig. 6(A) and (B). About $9 \mathrm{~s}$, later, the penetration front of water began to lead the front of rhodamine $B$ as shown in Fig. 7(A) and (B). For system B, as shown 


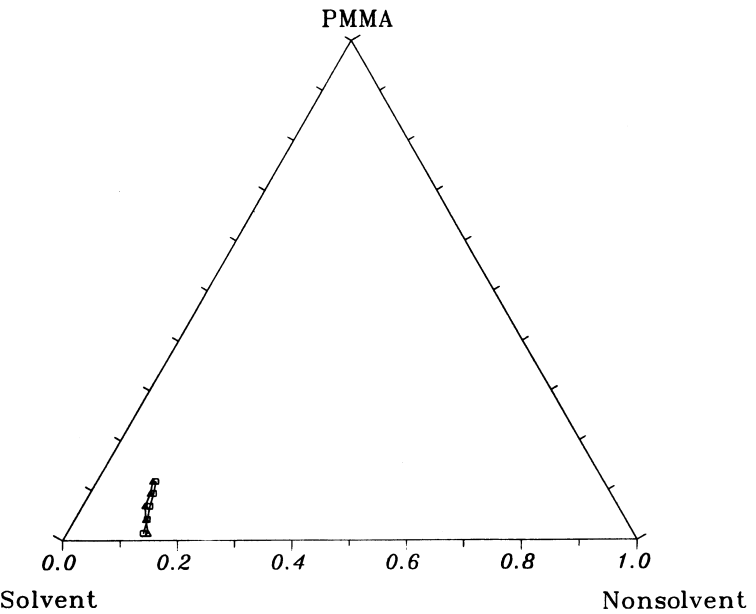

Fig. 5. Cloud-point curves for systems A and B: $(\Delta)$ system A; ( $\square)$ system B.

in Fig. 6(C) and (D), and Fig. 7(C) and (D), water can penetrate easily into the casting solution of PMMA/ acetone +Tween 80 , but rhodamine B (red color) can hardly get into the casting solution.

The distance from the tips of macrovoids to the coagulant-solution interface, representing the size of macrovoids, was determined by an image processing software for systems A and B. The dependence of this distance on time is plotted in Fig. 8(A). In addition, the data are replotted versus the square root of time in Fig. 8(B) to investigate the growth mechanism. For system $B$, the linear dependence of the position of the front of macrovoids on the square root of time was obtained. However, for system A, linear dependence of the growth front on the square root of time can only be obtained in the later stage of growth, when the growth front led the front of dye, but not in the initial stage, when the growth front and the dye font overlapped.

The linear dependence of the growth front on the square root of time indicates that the growth of macrovoids can be described by Fickian diffusion law. Hence, for system B, the growth mechanism of macrovoids is dominated by diffusion. Since the molecular weight of rhodamine B $(479 \mathrm{~g} / \mathrm{mol})$ is much larger than that of water, the diffusivity of rhodamine $B$ should be much smaller than water. Therefore, when the water dyed with rhodamine B was in contact with the casting solution, water could diffuse into the solution much faster than rhodamine B. The penetration of water into the casting solution could result in the formation of macrovoids and a dense layer at the solution-coagulant interface. The formation of a dense layer at the interface is quite common for the membranes with macrovoids [10]. This dense layer can inhibit the penetration of rhodamine B, and therefore, the red dye can hardly get into the casting solution. The above observation suggests that the formation of macrovoids in system B is due to diffusion.

For system A, diffusion seems not the dominant mechanism for the growth of macrovoids. If diffusion is the mechanism for the growth of macrovoids, the diffusion front of water should lead the front of rhodamine B because of the difference in diffusivity. The overlapping of these two fronts in the initial stage suggests that the growth of macrovoids in system A is not driven by diffusion but by some other mechanism which can make water and rhodamine B penetrate into the solution of PMMA/NMP with similar speed. Besides diffusion, convection was proposed to be a possible mechanism for the growth of macrovoids [5]. When the growth of macrovoids is driven by convection, the penetration fronts of water and rhodamine $\mathrm{B}$ would overlap. Hence, it seems that convection is a more appropriate mechanism than diffusion for the growth of macrovoids in system A. However, about $9 \mathrm{~s}$ after the contact of water with the solution of PMMA/ NMP, the front of water began to lead the front of rhodamine $\mathrm{B}$, indicating that diffusion began to dominate the growth of macrovoids. It should be noted that the size of macrovoids shown in Fig. 1(A) is about $100 \mu \mathrm{m}$ and the penetration depth is also about $100 \mu \mathrm{m}$ during the first $9 \mathrm{~s}$ of the contact of water with PMMA/NMP solution as shown in Fig. 8, indicating that the formation of macrovoids is almost completed in the first $9 \mathrm{~s}$, when the penetration is dominated by convective flow. Therefore, it seems reasonable to say that convection, instead of diffusion, is the dominant mechanism for the growth of macrovoids in system $\mathrm{A}$.

It should be noted that, during the observation of the penetration of coagulant into the casting solution, the casting solution was placed between two microscope slides. Such a model system might not be able to completely simulate the real membrane formation system. For example, the convective flow could be 


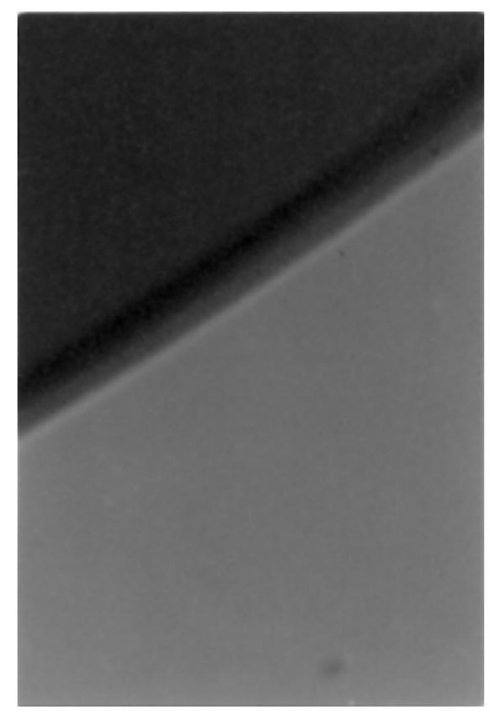

(a)

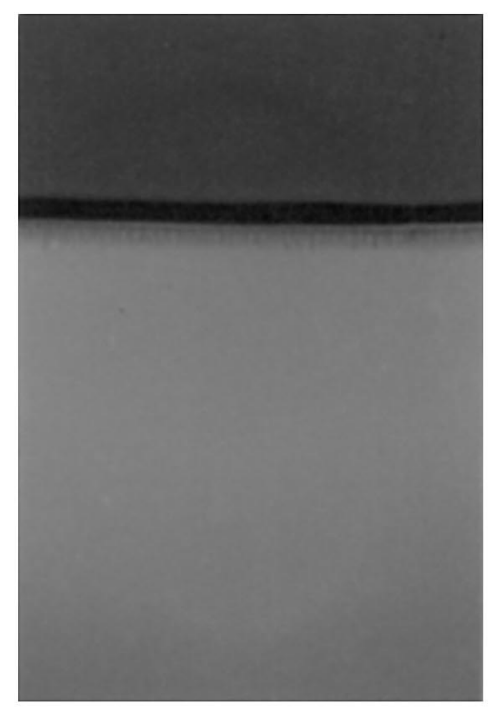

(c)

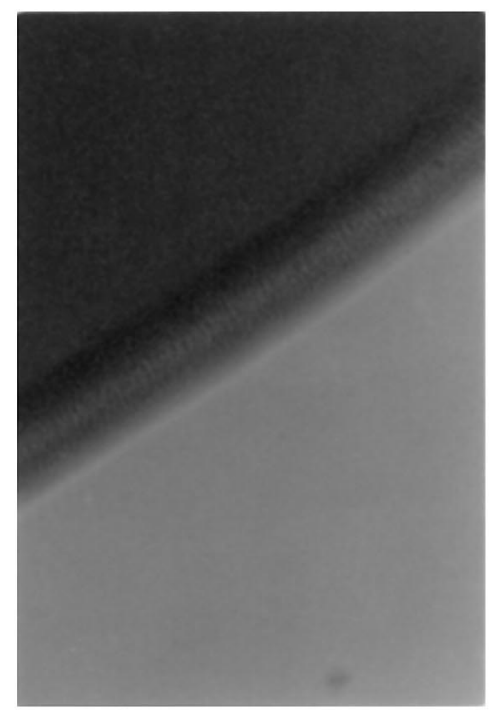

(b)

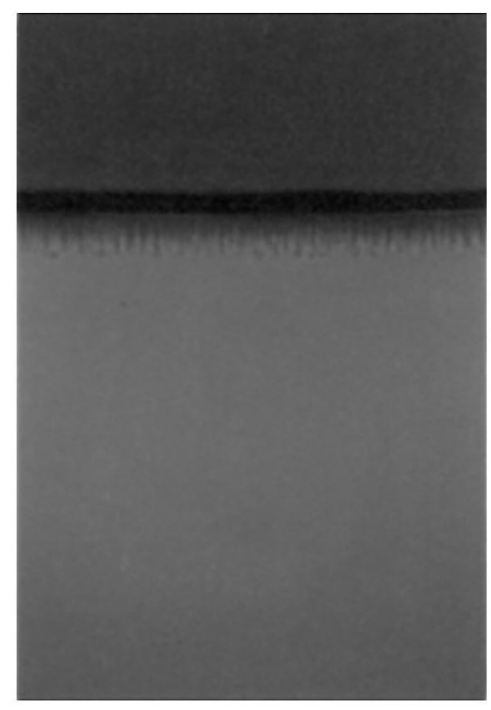

(d)

Fig. 6. Penetration of coagulant into casting solution for systems A and B (magnification $\times 100$ ). System A: (A) $3 \mathrm{~s}$; (B) $6 \mathrm{~s}$ and system B: (C) $3 \mathrm{~s}$; (D) $6 \mathrm{~s}$.

caused by the capillary effect between the two slides. Therefore, it seems reasonable to suspect that the penetration of rhodamine $\mathrm{B}$ into the solution of PMMA/NMP induced by convective flow might only be an artifact and does not exist in the real system. To prove that the above observation is not an artifact, the following experiment was performed.
One ml of PMMA/NMP solution was introduced by a pipette into a bath of water dyed with rhodamine $\mathrm{B}$, after about $10 \mathrm{~min}$, the precipitated polymer was removed from the water bath and dried in air. Same experiment procedures were performed again by introducing $1 \mathrm{ml}$ of PMMA/acetone+Tween 80 solution into the red water bath. For both systems, the surface 


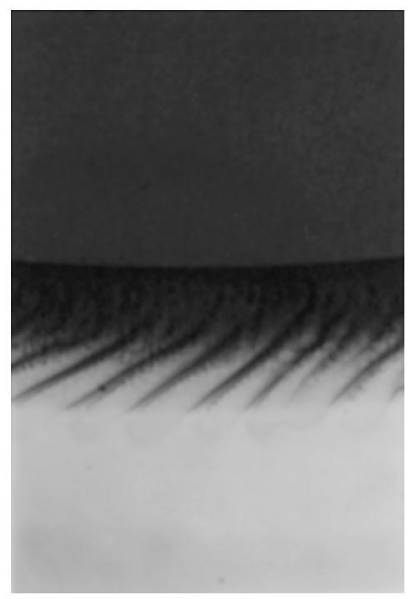

(a)

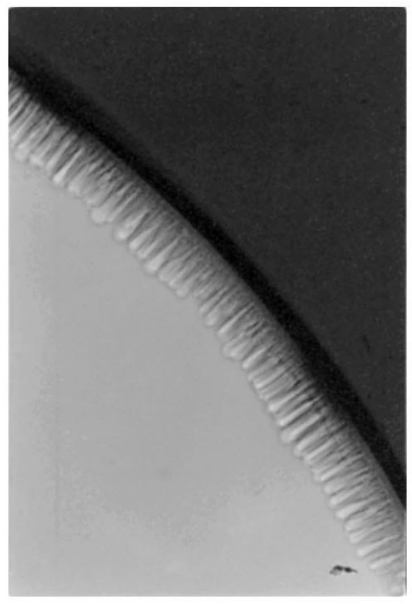

(c)

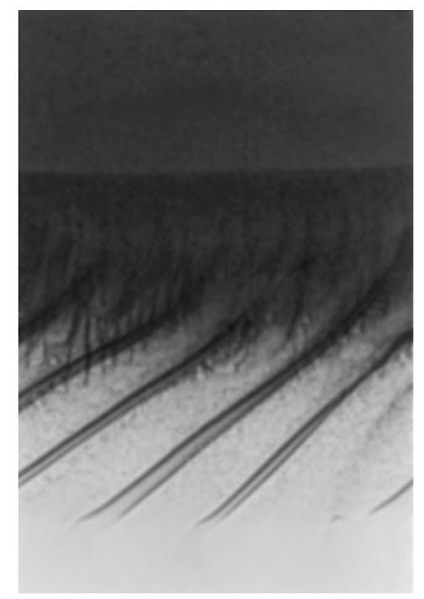

(b)

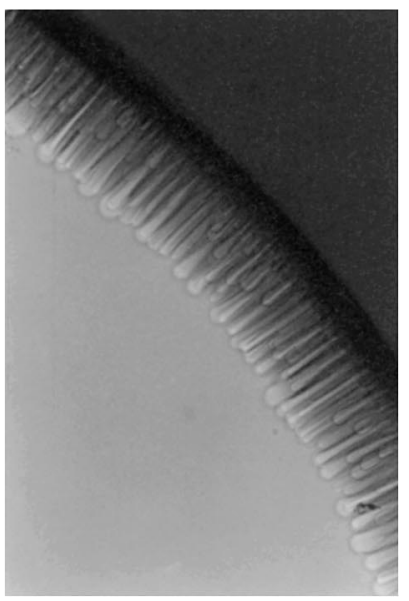

(d)

Fig. 7. Penetration of coagulant into casting solution for systems A and B (magnification $\times 100)$. System A: (A) $30 \mathrm{~s}$; (B) $60 \mathrm{~s}$ and system B: (C) $30 \mathrm{~s}$; (D) $60 \mathrm{~s}$.

of the precipitated polymer is reddish. However, the interiors of the precipitated polymer for the two systems have different color. For system A, it is red in the interior, indicating that rhodamine $\mathrm{B}$ can penetrate into the casting solution. For system B, the interior of the precipitated polymer for the other system is white, indicating that rhodamine B can hardly get into the casting solution. These observations agree well with those results from optical experiments. Since the experiment was performed by directly introducing the casting solution into a water bath without using any microscope slides, these obser- vations should be free from any artifacts resulted from microscope slides, suggesting that the conclusion obtained from the optical study is reliable and not artifacts.

In addition, on basis of the observation that the penetration speed of rhodamine B for system A is much larger than that for system $B$, it is expected that the membrane surface for system $B$ should be denser than that for system A. This expectation agrees well with the AFM analysis presented in Fig. 3: the polymer aggregation on the membrane surface is more complete for system B than for system A. The agree- 


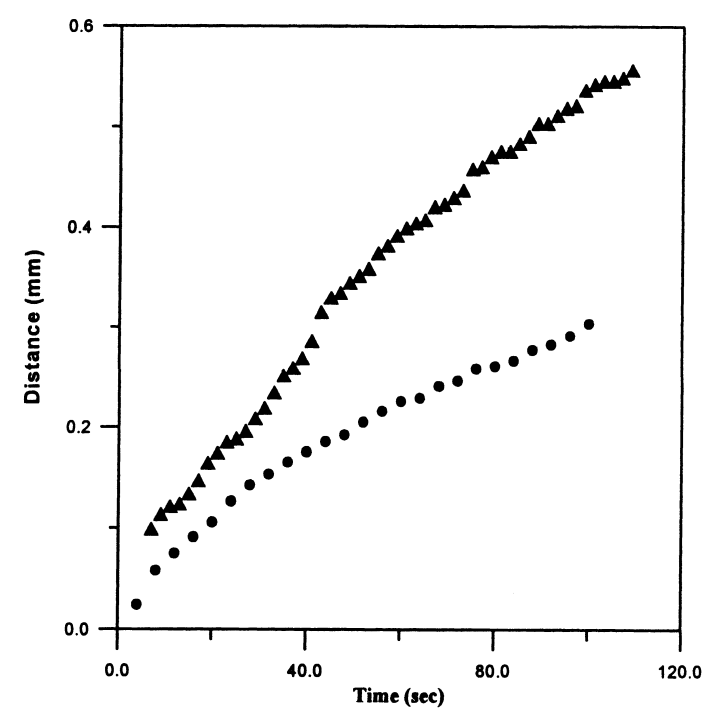

(A)

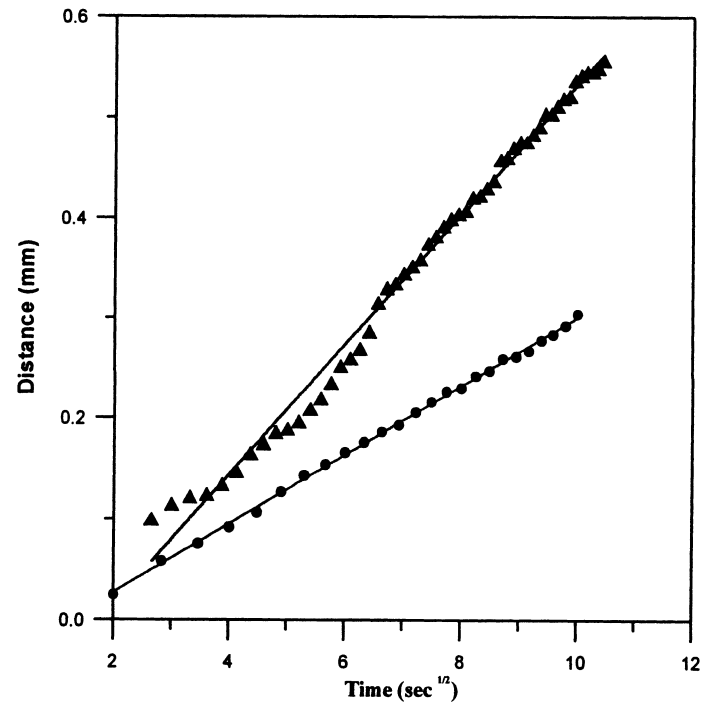

(B)

Fig. 8. Penetration distance of coagulant versus time for systems A and B: (A) system A; ( $)$ system B.

ment between the membrane surface structure and the optical observation can further support that the observation from the optical study is not an artifact.

\subsection{On the difference in the formation mechanism of macrovoids}

The difference in the microstructure between systems $\mathrm{A}$ and $\mathrm{B}$ described in Figs. 3 and 4 can be explained by the difference in the membrane formation mechanism. The initial penetration speed of coagulant into casting solution is higher for system A (convective flow) than for system B (diffusional flow) as shown in Fig. 6. The fast penetration of coagulant into the casting solution would result in fast precipitation, which would limit the movement of polymer chains and hinder the aggregation of polymer. Hence, the aggregation of polymer at the membrane surface is less complete for system A as shown in Fig. 3. The more complete aggregation of polymer for system B would then result in a denser skin which would limit the penetration of rhodamine $B$ as discussed in Section 3.2. The more complete polymer aggregation, resulted from slower penetration of coagulant, can also explain why system B possesses smoother microstructure than system A (Fig. 4).
Since the growth mechanism of macrovoids is different between systems $\mathrm{A}$ and $\mathrm{B}$, the method to inhibit the growth of macrovoids might be different. It was reported that the addition of salt in the coagulation bath is an effective method to inhibit the growth of macrovoids [16]. Experiments of adding salt in the water bath for systems A an B were performed. For system B, the addition of salt in the water bath can effectively prohibit the growth of macrovoids as expected (Fig. 9(C) and (D)). On the other hand, for system $\mathrm{A}$, the addition of salt cannot inhibit the growth of macrovoids as shown in Fig. 9(A) and (B). For system $B$ in which diffusion is the growth mechanism, the growth of macrovoids is due to the diffusion of polymer solvent into the growing macrovoids $[11,12]$. For such a system, the ability of the growing macrovoids to imbibe polymer solvent, strongly related to the composition in the growing macrovoids, is important in determining the size of macrovoids. Since the addition of salt can change the composition of the coagulant and then results in different composition in the growing macrovoids, it can successfully control the size of macrovoids. On the other hand, for system A, the addition of salt is not so effective because the growth of macrovoids is due to the convective flow, which is not sensitive to the composition of the coagulant. 


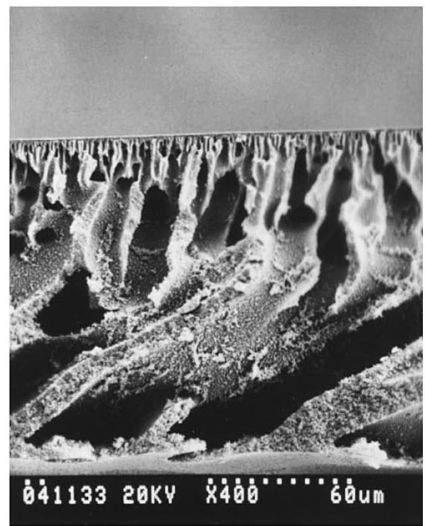

(a)

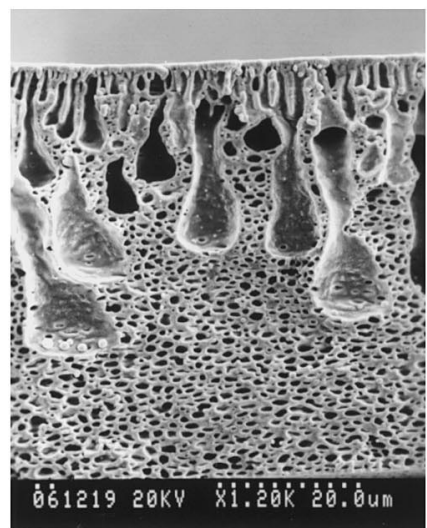

(c)

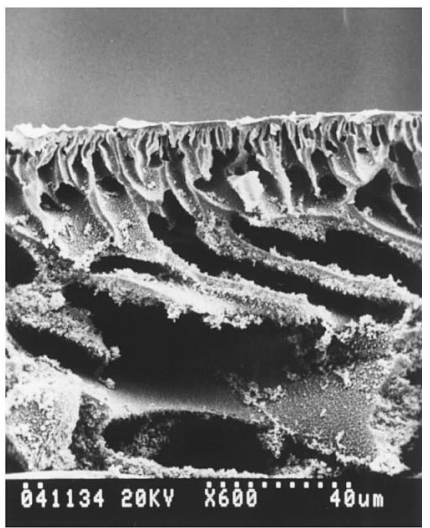

(b)

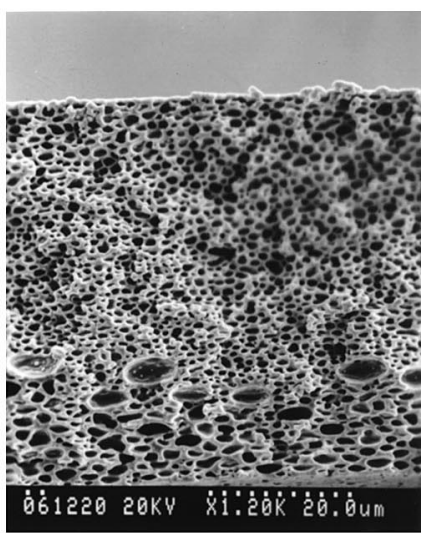

(d)

Fig. 9. Effect of adding salt in coagulation bath on membrane structure: (A) coagulant: $0.2 \mathrm{~g} / \mathrm{ml}$ saline solution, system A; (B) coagulant: $0.36 \mathrm{~g} / \mathrm{ml}$ saline solution, system A; (C) coagulant: $0.2 \mathrm{~g} / \mathrm{ml}$ saline solution, system B; (D) coagulant: $0.36 \mathrm{~g} / \mathrm{ml}$ saline solution, system B.

Another difference between systems A and B is their response to the change of casting thickness. The casting thickness was $300 \mu \mathrm{m}$ for the membranes discussed above. When the casting thickness was increased to $500 \mu \mathrm{m}$, no obvious change on the membrane structure was observed. However, when the casting thickness was increased to $2 \mathrm{~mm}$, the membranes fabricated by systems A and B possess different structures as shown in Fig. 10(A) and (B). For system A, the membrane (Fig. 10(A)) possesses a structure similar to that shown in Fig. 1(A) (casting thickness is $300 \mu \mathrm{m})$ but with larger membrane thickness. For system B, the structure of the membrane with a casting thickness of $2 \mathrm{~mm}$ (Fig. 10(B)) is different from that with a casting thickness of $300 \mu \mathrm{m}$
(Fig. 1(B)). Macrovoids only cover half of the membrane cross-section and do not extend over the whole membrane.

For the system dominated by diffusion (system B), since the growth of macrovoids is by imbibing polymer solvent into the nuclei of macrovoids $[11,12]$, the polymer solvent in the nuclei increases with increasing size of macrovoids, indicating a decrease in the driving force of the diffusion of polymer solvent into the nuclei during the growth of macrovoids. In other words, the ability of the growing macrovoids to imbibe the polymer solvent during the growth of macrovoids decreases with the increasing size of macrovoids. Therefore, the macrovoids cannot grow large enough to cover the whole membrane when the casting solu- 


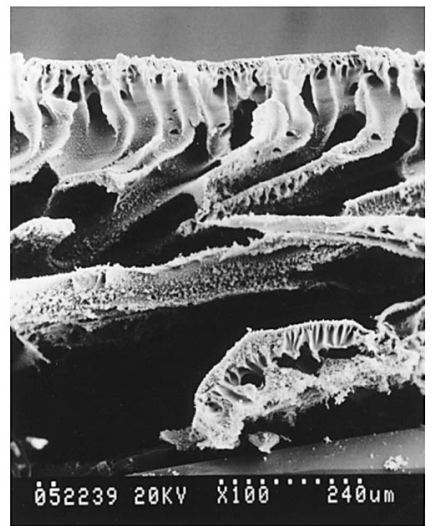

(a)

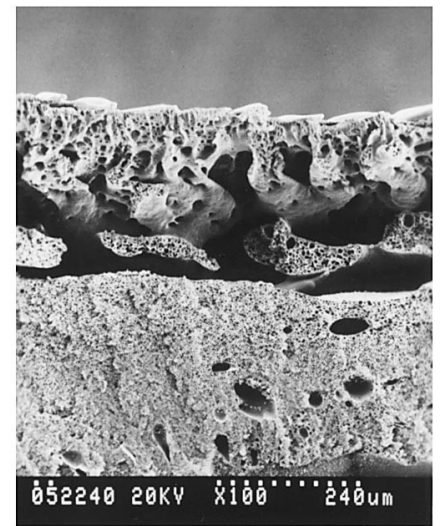

(b)

Fig. 10. Effect of casting thickness on membrane structures (casting thickness: 2 mm): (A) system A; (B) system B.

tion was increased to $2 \mathrm{~mm}$. On the other hand, for system A, the growth of macrovoids is due to the convective flow and the composition in the growing macrovoid does not change during growth; therefore, the macrovoids are easy to grow to the whole crosssection of the membrane. The difference in the growth mechanism could explain the difference between systems $\mathrm{A}$ and $\mathrm{B}$ in the effect of increasing casting thickness.

\subsection{Diffusion and convection}

In the present work, we presented experimental data suggesting that convection and diffusion can both cause growth of macrovoids. Understanding why the formation mechanism of macrovoids is different between systems A and B is important for constructing the complete mechanism of the formation of macrovoids. More investigations are required to provide an answer for this question. In the following, a possible answer is suggested. It was observed that, when the casting solutions of systems A and B were in contact with water, the surface of the casting solution of system A vitrified faster than that of system B. The fast vitrification might cause the shrinkage of polymer matrix and result in convective flow [5], responsible for the growth of macrovoids in system A. For system $\mathrm{B}$, the addition of surfactant Tween 80 might be able to plasticize the polymer, and the vitrification speed is therefore slower. Since the vitrification speed is not fast enough to cause convective flow, the growth of macrovoids in system B is caused by diffusion. To verify the above deduction, more investigations are required.

\section{Conclusion}

In this work, formation of macrovoids was investigated in two membrane formation systems: PMMA/ NMP/water (system A) and PMMA/acetone+Tween 80/water (system B). The results indicate that, although the macrostructures of the membranes fabricated by systems A and B are similar, the microstructures are different. The difference in the microstructure can be explained by the difference in the penetration speed of coagulant into the casting solution. The results also indicate that the growth of macrovoids is by convection for system $\mathrm{A}$ and by diffusion for system B. The difference in the growth mechanism of macrovoids can also be used to explain some other differences between systems $\mathrm{A}$ and $\mathrm{B}$ : system $B$ is more sensitive to the casting thickness than system A, and the addition of salt in the coagulation bath can effectively inhibit the growth of macrovoids in system B but not in system A.

\section{Acknowledgements}

The authors wish to sincerely thank the National Science Council of Taiwan, ROC(NSC 87-2216-E- 
033-004 and NSC 87-2214-E-033-008) for the financial support.

\section{References}

[1] C.A. Smolders, A.J. Reuvers, R.M. Boom, I.M. Wienk, Microstructures in phase-inversion membranes. Part 1: Formation of macrovoids, J. Membr. Sci. 73 (1992) 259-275.

[2] M.A. Frommer, R.M. Messalem, Mechanism of membrane formation. VI. Convective flows and large void formation during membrane precipitation, Ind. Eng. Chem. Prod. Res. Develop. 12 (1973) 328-333.

[3] S.H. Herbig, J.R. Carinal, R.W. Korsmeyer, K.L. Smith, Asymmetric membrane tablet coating for osmotic drug delivery, J. Control. Release 35 (1995) 127-136.

[4] D.M. Wang, F.C. Lin, L.Y. Chen, J.Y. Lai, Application of asymmetric TPX membranes to transdermal delivery of nitroglycerin, J. Control. Release 50 (1998) 187-195.

[5] H. Strathmann, K. Kock, P. Amar, The formation mechanism of asymmetric membranes, Desalination 16 (1975) 179-203.

[6] R. Matz, The structure of cellulose acetate membrane I. The development of porous structures in anisotropic membranes, Desalination 10 (1972) 1-15.

[7] P. Neogi, Mechanism of pore formation in reverse osmosis membranes during the casting process, AIChE J. 29 (1983) 402-410.

[8] R.J. Ray, W.B. Krantz, R.L. Sani, Linear stability theory model for finger formation in asymmetric membranes, J. Membr. Sci. 23 (1985) 155-182.
[9] Y. Termonia, Fundamentals of polymer coagulation, J. Polym. Sci.: Polym. Phys. Ed. 33 (1995) 279-288.

[10] A.J. Reuvers, Membrane formation: Diffusion induced demixing processes in ternary systems, Ph.D. Thesis, Twente University of Technology, Netherlands, 1987.

[11] L. Broens, F.W. Altena, C.A. Smolders, D.M. Koenhen, Asymmetric membrane structure as a result of phase separation phenomena, Desalination 32 (1980) 33-45.

[12] S.A. McKelvey, W.J. Koros, Phase separation, vitrification, and the manifestation of macrovoids in polymeric asymmetric membranes, J. Membr. Sci. 112 (1996) 29-39.

[13] F.G. Paulsen, S.S. Shojaie, W.B. Krantz, Effect of evaporation step on macrovoid formation in wet-cast polymeric membranes, J. Membr. Sci. 91 (1994) 265-282.

[14] J.M. Cheng, D.M. Wang, F.C. Lin, J.Y. Lai, Formation and gas flux of asymmetric PMMA membranes, J. Membr. Sci. 109 (1996) 93-107.

[15] F.C. Lin, D.M. Wang, C.L. Lai, J.Y. Lai, Effect of surfactants on the structure of PMMA membranes, J. Membr. Sci. 123 (1997) 281-291.

[16] Y. Termonia, Molecular modeling of phase-inversion membranes: effect of additives in the coagulant, J. Membr. Sci. 104 (1995) 173-180.

[17] M. Mulder, Basic principle of membrane technology, Kluwer Academic Publishers, London, 1991.

[18] R. Koningsveld, Liquid-liquid equilibria in quasi-ternary systems, Chem. Zvesti 26 (1972) 263-287.

[19] F.-J. Tsai, J.M. Torkelson, Roles of phase separation mechanism and coarsening in the formation of poly(methyl methacrylate) asymmetric membranes, Macromolecules 23 (1990) 775-784. 